\title{
PERMANENCIA DEL DETERMINISMO GEOGRAFICO EN LA ENSEÑANZA DE LA GEOGRAFIA EN COLOMBIA*
}

Ovidio Delgado Mahecha ${ }^{* *}$

\section{INTRODUCCION}

En Colombia la geografía es más conocida como asignatura escolar que como disciplina científica. La gran mayoría de los colombianos tiene de la geografía la concepción aprendida en su tránsito por la escuela primaria y secundaria, que la considera no como una ciencia, sino como una cualidad de las regiones; como-el conjunto de las condiciones físicas de las mismas (relieve, clima, hidrografía, etc.) que les dan identidad y que explican por sí mismas las desigualdades regionales en sus aspectos humanos. Tal enfoque de la enseñanza coincide con los postulados básicos del determinismo geográfico que son muy frecuentes en los libros de texto.

El presente ensayo pretende:

1. Detectar las relaciones existentes entre el determinismo geográfico y los contenidos de la enseñanza de la geografía en Colombia.

2. Identificar la presencia de postulados deterministas en textos escolares empleados para la enseñanza de la geografía.

3. Establecer las tendencias de permanencia o de cambio de las ideas del determinismo geográfico en la enseñanza de la geografía en Colombia.

\section{El determinismo geográfico}

De acuerdo con Lewthwaite (1966) el determinismo geográfico es una variante del determinismo que considera que el medio geográfico constituye el principal control de la vida humana. No es sinónimo de ambientalismo, bajos cuyos conceptos la geografía se define como el estudio de las interreaciones entre el hombre y su medio. Según Estébanez (1982: 50), "el determinismo geográfico como forma concreta del determinismo en general, supone que la libertad del hombre está dirigida en grado de mayor o menor necesidad por factores del medio físico, que ejercen una influencia en la constitución física y moral del hombre individual y social". Es decir, que todas las actitudes y actividades del hombre están gobernadas por las condiciones físicas del medio natural que lo alberga.

Como modelo de explicación científica en geografía, el determinismo se destaca desde finales del siglo XIX (Claval, 1974). Pero las cuestiones relacionadas con el dominio ambiental sobre el hombre y la sociedad, tienen un largo pasado (Isachenko 1971). Varios autores (James, 1972; Isachenko, 1971; Estébanez, 1982; Vilá, 1983) encuentran sus raíces en la antigüedad, desde Hipócrates, Aristóteles, Tucídides, Jenofonte, Herodoto, Polibius y Estrabón, quienes de una manera u otra acudieron a las condiciones naturales como el clima y la posición geográfica excepcionales, para explicar y justificar el dominio de unos pueblos sobre otros. En el período feudal (Isachenko, 1971) hombres de ciencia

\footnotetext{
${ }^{*}$ El autor expresa sus agradecimientos al Doctor H.F. Rucinque director del EPG por su asesoría en la realización de este trabajo.

** Ovidio Delgado Mahecha es Licenciado en Ciencias Sociales. Geógrafo. Director del Departamento de Ciencias Sociales de la Universidad Pedagógica Nacional.
} 
como Bacon y Albertus Magnus, quienes se hallaban familiarizados con los trabajos de los autores antiguos, hicieron tímidas aseveraciones sobre el impacto del medio natural sobre el hombre; pero la ideología religiosa excluía toda aceptación del determinismo geográfico que pudiera de alguna forma compartir con Dios su poder sobre el hombre.

El determinismo geográfico revive cuando la burguesía emerge como clase política, criticando la idea de determinación divina, para lo cual se basó en los autores clásicos de la antigüedad. Ejemplos de este renacer pueden verse en Montesquieu y muchos otros filósofos (Isachenko 1971). Así, el filósofo Johan Gotfried Herder encuentra en las diferencias medioambientales la razón de las diferencias culturales entre Europa y otras regiones del mundo, y considera que la civilización europea debe su prosperidad y su brillante situación en el universo de los pueblos, a un concurso de circunstancias como la multiplicidad de pueblos e ideales, el clima templado y otros factores físicos que le dan superioridad y le han permitido alcanzar una cultura humana activa (Adamov, 1983).

Otro filósofo, Víctor Cousin, proclamaba lo siguiente, según citas de Lewthwaite (1966:9) y Vílá Valentí (1983: 197):

"Si, señores, dadme el mapa de un país, su configuración, su clima, sus aguas, sus vientos y toda su geografía física; dad me sus productos naturales, su flora, su fauna y yo me encargo de decíros de antemano cómo será el habitante de dicho país y cuál será el papel que éste desempeñará en la historia. Y ello no accidental, sino necesariamente, no tal época, sino en todas".

En el campo específico de la geografía moderna, es a Karl Ritter a quien se le asigna la responsabilidad de haber introducido el determinismo como explicación geográfica, en su intento de estudiar las relaciones entre la superficie terrestre y la actividad humana (James 1972;Claval, 1974; Estébanez, 1 982; Gómez Mendoza et al, 1982). En 1850 Ritter expresó que:

"El hecho de que los continentes tengan superficies diferentes explica el poderío de los pueblos y la posibilidad que les es dada de dominarlas. El aparente azar que preside la disposición relativa de las masas de tierra refleja una ley cósmica superior que ha determinado necesariamente todo el proceso de desarrollo de la humanidad. La separación a primera vista puramente física del Viejo y del Nuevo Mundo, de los continentes y de las islas resulta ser la esencia de la relación espacial universal. La desigual distribución de los dones naturales es el estimulante fundamental del desarrollo de los intercambios universales. La débil superficie de Europa y la armonía de sus formas limitadas es la condición de su libertad y de su capacidad de dominación" (Cita traducida por Gómez Mendoza eta! 1983: 177).

Del mismo tenor, aunque más radicales, son las aseveraciones de Arnold Guyot, discípulo de Ritter, quien en su obra The Earth and Man, publicada en Boston en 1852, intenta explicar lo que él llama "la marcha geográfica de la historia", con fundamentos del determinismo ambiental, remitidos a Dios como causa final. Considera que el desarrollo desigual entre los países del norte y del sur es un hecho histórico, cuyas causas son unas de naturaleza moral y otras de naturaleza física, las cuales han determinado que los continentes del sur no participen en el desarrollo de la humanidad. Guyot (1 852: 33), refiriéndose a los continentes del sur concluye: 
"Las razas habitantes de ellos están cautivas en los lazos de toda la fuerza natural. Ellos nunca podrán echar abajo las cercas que los separan de nosotros. Esto es para nosotros las razas favorecidas. Vamos a ellos. La naturaleza tropical no podrá ser conquistada y dominada, excepto por hombres civilizados, armados con toda la fuerza de la disciplina, inteligencia y experiencia industrial. Esto es, entonces, que desde los continentes del norte, ellos los del sur, esperen su liberación; esto es, por la ayuda que los hombres civilizados de los continentes templados se dignen darle a los hombres de las tierras tropicales para ingresar al movimiento del progreso universal y el perfeccionamiento".

Como se puede apreciar, en Ritter y en Guyot confluyen el determinismo y ja geopolítica, aunque sus conclusiones derivan más de una causalidad basada en la fé que de una comprobación científica, pues su enfoque teleológico siempre los remite a Dios como causa final. Uno no puede encontrar en las palabras de Guyot otra cosa que la justificación del colonialismo y la recomendación del mismo. Guyot se adelantó bastante tiempo a Ratzel en pretender dar una base científica a la expansión territorial de los estados más fuertes.

Es en el siglo XIX, cuando la geografía acoge formalmente el modelo newtoniano de explicación, que ya había probado en las ciencias naturales la eficacia de la relación determinista causa-efecto (Harvey 1969:98) considera que "la principal contribución de Humboldt y Ritter a la explicación en geografía, fue su insistencia en afirmar que podían establecerse leyes de causa y efecto que explicasen las distribuciones geográficas. De forma que la noción de causa y efecto se convirtió en una de las formas dominantes de explicación en geografía durante el siglo XIX". Así, la geografía tendría un lugar garantizado en el paradigma científico imperante. En efecto, como lo confirma Harvey (1969: 427).

"La forma de conceptuar mecanismos y procesos que predominaba al final del siglo XIX era determinista o como se llama a veces, mecanicista. No hay duda de que esta concepción del mundo se inspira mucho en ja mecánica de Newton en la que los movimientos de los planetas podían predecirse sin ninguna incertidumbre a partir de un conjunto fundamental de ecuaciones diferenciales".

Pero si bien es cierto que todas las ciencias, incluida la geografía, se concibieron según el modelo determinista de las ciencias físicas, es necesario reconocer que se tenía cierta prudencia cuando se abordaba el tema del hombre, que por otra parte ya se había incorporado como objeto temático de la ciencia que debería explicarlo como individuo y como ser social. La solución llegó con la obra de Darwin (1 859) sobre el origen de las especies que "incidió de forma decisiva en el panorama general del conocimiento decimonónico y en concreto en el del conocimiento geográfico"... y que, "proponía un modelo particularmente riguroso y expresamente científico para abordar coherentemente el tratamiento de las nuevas positividades decimonónicas referentes a la historicidad y a la incorporación del hombre y de sus relaciones con la naturaleza de los objetos del conocimiento positivo" (Gómez Mendoza et al 1982: 31).

Según D.R. Stoddart (1966) la obra de Darwin aporta a la geografía los puntos siguientes:

1. El concepto de desarrollo a través del tiempo, o la evolución. Este concepto influyó notoriamente en la obra de WM. Davis, particularmente en la idea de evolución de ríos 
y paisajes, expresada en estados de juventud, madurez y vejez, planteamiento mejor conocido como el modelo del ciclo geográfico.

2. El concepto de relaciones entre un organismo y su habitat, que permite analizar los organismos en términos de los roles que juegan en su medio ambiente particular, en donde las condiciones físicas de vida constituyen una unidad orgánica junto con las relaciones cerradas existentes entre todos los organismos. Esto se refleja en el concepto vidaliano de región y en los enfoques ecológicos de la geografía que hacen énfasis en las relaciones hombre-tierra.

3. El concepto de selección y lucha, o de competencia que está asociado con el laissezfaire económico y político, y que tuvo sus efectos en geografía particularmente en la obra de Ratzel, quien formuló a partir de esta noción sus leyes para el desarrollo de los estados, y justificó las luchas entre naciones como luchas por su espacio vital.

4. Los conceptos de variación aleatoria, que no fueron tenidos en cuenta por los geógrafos deterministas, pero que actualmente han sido retomados por los enfoques probabilistas.

Puede decirse que en la obra de Darwin tanto la geografía física como la geografía humana creyeron encontrar un asidero científico para el determinismo geográfico. Al igual que todas las ciencias naturales y sociales, la geografía no pudo mantenerse al margen de la revolución científica generada por la obra de Darwin y como dice Harvey (1969: 417).

"Hacia finales del siglo, Davís y Ratzel habían adoptado posiciones evolucionistas en sus estudios de las formas y de la difusión del paisaje, respectivamente, una postura evolucionista que llegó a dominar la geografía durante medio siglo y que tuvo un profundo impacto en la geografía humana con los trabajos de Huntington, Taylor, Sauer y otros".

Sin embargo, según Stoddart (1966) los geógrafos no asimilaron bien la obra de Darwin, pues si tomaron el concepto de evolución, dejaron de lado la noción de variación aleatoria y cayeron sin remedio en el determinismo geográfico, apoyándose también falsamente en una mala interpretación del principio de relaciones entre los organismos y su habitat, que Darwin consideraba no como relaciones unilaterales y deterministas, sino como interrelaciones. A propósito de lo anterior dice Stoddart (1966: 688) que "sin embargo los geógrafos interpretaron la revolución biológica en términos de cambio a través del tiempo: lo que para Darwin fue un proceso, para Davis y otros era una historia".

Ya se dijo que tanto la geografía física como la humana son deterministas. Pero es en la naciente geografía humana en donde el impacto del determinismo fue mayor, pues al fin y al cabo la geografía física como ciencia de la tierra no se afectaba en alto grado al adoptar una vez más los modelos explicativos de las ciencias naturales afines a ella.

Con Ratzel, quien combina el darwinismo con el evolucionismo de Spencer, se inicia una tendencia en la geografía humana que fue continuada con ahínco por su discípula Ellen Churchill Semple y Ellsworth Huntington, la primera haciendo énfasis en la localización, y el segundo en la influencia de los cambios climáticos en la historia humana. Esta tendencia es lo que Estébanez (1982) llama paradigma determinista en el cual todo se explica acudiendo al medio ambiente como causa final. Ratzel, según cita de Gómez Mendoza et a! (1982:38) afirma que "los procesos humanos, sociales y políticos que 
tienen lugar en el espacio geográfico responden a esquemas de determinación causal en los que las condiciones naturales desempeñan un papel incuestionablemente decisivo".

Ellen Churchill Semple — de quien Broek (1965) dice que su preeminencia se debió a que expresó claramente los puntos de vista de sus colegas norteamericanos- considera que el hombre es un producto de la tierra que está determinado por ella en todos sus aspectos fisiológicos, espirituales y morales (James 1972).

Huntington (1 940) presenta un modelo de explicación para la geografía humana en el que las condiciones físicas determinan todas las condiciones de la vida, al mismo tiempo que controlan las actividades del hombre desde la satisfacción de las necesidades materiales, hasta las formas de gobierno, el arte y la religión. Concluye que el medio ambiente determina la civilización y el progreso de la humanidad.

Esencialmente el determinismo geográfico se fundamenta en el uso seudocientífico de la explicación causal. Se trata de un intento por analizar en forma unidireccional un conjunto de interacciones complejas (Harvey 1969).

\section{El determinismo geográfico en los textos de enseñanza}

En las escuelas y colegios de Colombia los estudiantes reciben lecciones de geografía dos o tres veces por semana. Allí se informan sobre su país y sobre otros mundos que no pueden percibir directamente y que al decir de Vilá Valentí (1983) puede llamarse una "geografía exótica". Y un poco de lo que allí reciben es su saber geográfico, casi siempre el de toda su vida, y su concepción de la geografía.

La geografía escolar colombiana puede ser analizada a través de los textos utilizados, toda vez que ellos constituyen prácticamente los únicos materiales a disposición del estudiante.

Sin pretender desconocer la urgencia y la necesidad de un análisis integral de la enseñanza de la geografía, aquí, como ya se dijo, solamente se demostrará que los principios del determinismo geográfico son una constante de amplio espectro en los libros de texto que desarrollan fielmente los programas oficiales. Textos y programas con sus vicios y deficiencias han permanecido sin variaciones de fondo a través de muchos años.

En la década contada a partir de 1950 los estudiantes de los cursos de Geografía de Colombia aprendieron los más granados fundamentos del determinismo decimonónico. Aprendieron que:

"Los pueblos que disfrutan de más favorable situación geográfica son los de latitudes medias, donde las lluvias son frecuentes y las temperaturas no son, en general ni muy altas ni muy bajas. $Y$ si por contera disponen de tierras fértiles y de relieve apenas ondulado, y cuentan con un subsuelo rico en minerales, constituyen el prototipo de las "zonas de atracción", las más aptas para el progreso de las sociedades. Tal ocurre en Estados Unidos y Europa" (Franco, 1952: 221).

Y también se enseñó que el monocultivo cafetero en Colombia era consecuencia única de las condiciones físicas del país: 
"¿Por qué se ha orientado nuestra agricultura hacia el café con tanta unilateralidad? ¿Por qué nos hemos especializado en la industria cafetera en tan gran escala? No es por capricho, ni por vocación: es por la fuerza del determinismo geográfico" (Franco, 1952: 221).

El autor de las dos citas anteriores, cuyo libro de texto continúa vigente con pocas modificaciones, fue en su momento un distinguido educador, inspector de educación nacional y miembro de la Sociedad Geográfica de Colombia (Franco 1952: 3).

En 1958 apareció la séptima edición de la Geografía Superior de Colombia de Justo Ramón. Allí el autor incorpora en su texto como lectura complementaria un discurso académico de Daniel Samper Ortega, que le sirve para ilustrar la importancia de las montañas colombianas, que además de determinar el clima y las características del hombre colombiano, también son responsables de nuestro sistema político. Así se expresa Samper, citado y aceptado por Justo Ramón (1958: 37-38):

"La topografía colombiana es, pues, en gran parte responsable de nuestra fisonomía legalista y democrática; somos un país donde el caudillo no arraiga, porque tendría que dominar el territorio, arruga por arruga, desde Santander hasta Nariño; mas en cada arruga de aquellas en que prosperan las poblaciones, separadas unas de otras por quiebras profundas, los hombres tienen distintos intereses y distintas simpatías. El prestigio del caudillo es como un viento, que solo orea en las llanuras abiertas; nuestros únicos caudillos nacieron en el Cauca, el ancho Cauca de valles dilatados. Pero su prestigio se adelgazó al volar de cañada en cañada, como se adelgazan y evaporan las nubes que nos penetran desde el mar.

Sin embargo, a pesar de sus similitudes, nuestras cordilleras son distintas y han educado de diversas maneras a los hombres que se abrigan entre sus vegas y aristas. La cordillera oriental abunda en valores plácidos, y esa placidez se ha infiltrado en el cundinamarqués y el boyacense, que amarran su cuerpo a la tierra y sueltan a volar la fantasía. Los hombres del Reino somos indolentes, aún los santandereanos, a quienes el valor y la osadía no restan apeño al terruño y al hogar.

En Colombia, gracias a las montañas, a estas montañas que a la vez nos separan y nos unen, hemos tenido la fortuna de no vivir girando en torno a un punto único. Cada región posee un centro de suficiente entidad para que la vida provinciana tenga un sentido y un valor. Las dictaduras son fáciles allí donde basta a un hombre imponerse en la capital, pero son imposibles en Colombia, donde las ciudades de provincia tienen idéntico influjo en nuestro destino y gozan de idéntico prestigio en el país".

Otro autor de textos de geografía es Federico Arbeláez Lema, quien en su libro Geografía Universa/Americana, cuya segunda edición se publicó en 1967, explica las diferencias sociales, económicas y culturales entre América del Norte y América del Sur, acudiendo a las condiciones físicas como causa fundamental. Su libro fue publicado también en 1977, con los mismos contenidos por una editorial diferente a la de 1967. El autor no es geógrafo; es doctor en filosofía, letras y pedagogía, con estudios de postgrado en las universidades de Birminghan, Londres y La Soborna. Estudió administración pública en la ESAP; profesor de Bachillerato y universidades, consultor de la UNESCO, inspector nacional de educación, y otros estudios y cargos más que conforman su hoja de 
vida impresa en la contracarátula del libro. Dice así el doctor Arbeláez (1967:20; 1977: 28) refiriéndose a las diferencias entre las regiones americanas:

"A pesar de las grandes semejanzas, las diferencias son muchas y han influido en la historia, los géneros de vida y La economía de cada una de las partes en que se divide América.

La situación es la mayor diferencia. Mientras Norteamérica se encuentra casi toda en el hemisferio norte, de climas templados y fríos, y más próxima a las masas terrestres, Centroamérica está toda en la zona de latitudes bajas y Suramérica en parte, pasando por ella la línea ecuatorial; solamente el sur de nuestro continente está en la zona templada meridional.

Así, América del Norte disfruta de una posición privilegiada, en tanto que América del Sur es el área continental aislada de la tierra. Esto explica las diferencias humanas y económicas de las Américas".

Los planteamientos del autor en referencia nos hacen recordar los argumentos de Ritter y Guyot a mediados del siglo XIX, pero esta vez en la pluma de un hombre de latitudes bajas que cien años más tarde los acepta como verdades absolutas.

En 1968, con el texto Geografía Superior de Colombia, los profesores Javier Ocampo López y Ramón Franco R. ocuparon el primer puesto en un concurso nacional de obras didácticas y se hicieron acreedores al Premio Félix de Bedout. El libro en mención, es una trascripción literal de la obra Colombia, Geografía Superior Económica publicada por Ramón Franco en 1952 pero enriquecida con datos estadísticos más actualizados y con algunas consideraciones sobre la desventajosa posición geográfica de nuestro país con respecto a los países de la zona templada, y las mejores condiciones de Colombia frente a otras naciones africanas y asiáticas que comparten con ella las latitudes bajas, pero que no tienen montañas como las nuestras, Ocampo López y Franco R. (1968: 82) dicen:

"Colombia, en plena zona tórrida, debiera tener un clima amazónico como el de Liberia, como el del Congo Belga, o como el de Sudán, Guineo y Nigeria en el Corazón de África, o como el de Sumatra y Borneo, regiones ardientes e inhospitalarias, hoy por hoy, para el desarrollo de una civilización. Si estuviéramos a merced de la latitud, de manera exclusiva y total, Colombia sería el imperio de la manigua y muy remotas serian nuestras esperanzas de servir de asiento a una cultura. Pero la arrugada configuración de los Andes, nos ibera de ese determinante geográfico y nos brinda todos los climas de la tierra. De modo que el relieve y la topografía de nuestro suelo, corrigen y moderan el clima ardoroso y nos dan puntos de escala para ir avanzando en la incorporación de la región tropical a la vida y a la historia antropogeográfica".

La obra que venimos comentando alcanzó la octava edición en 1977 y hoy está a disposición de maestros y estudiantes. Después de treinta años de aparecida la primera edición, su estructura permanece intacta, con los mismos contenidos pero con un enfoque determinista aún más acendrado. La última edición del texto es de 1982 y aparece suscrita por Ramón Franco R. y Fabiola Franco B., con el título de Geografía Económica de Colombia, en donde después de sugerir a los profesores de geografía que "la geografía patria tiene una misión de singular importancia: dar un sólido criterio sobre lo que es el país, cuáles son sus posibilidades, cuál es su destino histórico y hacia dónde y 
cómo debe orientarse su progreso" (Franco R, Franco B., 1982:5) los autores expresan lo siguiente (1. 1982: 51):

"El clima es el factor más influyente en la historia de las diversas razas humanas. Su acción es directa, por medio de la presión atmosférica, electrización, humedad, paisaje. E indirecta, por razón del mundo vegetal y animal y los alimentos que ellos proveen.

Cada clima tiende a producir efectos síquicos propios, que son innatos en los indígenas y adquiridos en los inmigrantes. Se observa una relación directa entre el vigor mental y la variedad climática (climas de latitudes medías). Los pueblos de estos climas dominan a los de los trópicos por su mayor energía e iniciativa.

En climas enervantes las necesidades de la vida se reducen a un mínimo, por las dificultades y el poco esfuerzo de trabajo. Y no propician la cooperación de los hombres para alcanzar fines útiles a todos, que es lo que constituye el móvil del progreso social.

En las zonas templadas o de medias latitudes, la vida no es tan cómoda que degenere al linaje humano, ni tan severa que todas las energías se absorban en la tarea de mantener unidos cuerpo y alma".

Si nos atenemos a las recomendaciones sobre el papel de la geografía y a las conclusiones de los autores, no nos queda otro remedio que aceptar que "lo que es el País", es un pueblo tropical y que su "destino histórico" es ser dominado por pueblos de latitudes medias, porque "los pueblos de estos climas dominan a los de los trópicos por su mayor energía e iniciativa". Triste misión la de la geografía y sus maestros.

En Colombia la producción de textos de geografía es abundante; pero todos los manuales carecen de un tratamiento científico, ya que por una parte sus autores no son geógrafos y en muchos casos son especialistas en otras disciplinas, que escriben a la vez sobre geografía, didáctica de la matemática, didáctica del álgebra, la geometría y la trigonometría, como es el caso de Ramón Franco, quien deja constancia de sus obras en la contracarátula de su último texto. Por otra, los textos han sido reimpresos año por año, sin ser adaptados a las modernas concepciones de la geografía, y unos han servido de base para otros, sin ser sometidos a la más leve crítica. Para el caso que nos ocupa, las explicaciones deterministas, al igual que en los textos ya citados, son un común denominador en los que a continuación se comentan, todos ellos de amplio uso en la educación secundaria.

Allí, el clima aparece como factor determinante de la superioridad de los pueblos de la zona templada, del carácter de los individuos y de las comunidades, de la pasión del hombre por el trabajo o por la haraganería, de la alimentación y de las actividades económicas. El hombre aparece como un agente que se adapta paulatinamente a las condiciones del medio que le fija los límites de su actividad económica y cultural y que lo marca y le imprime un carácter indeleble, no solamente en su tipo de vivienda o en su vestido, sino también, en sus sentimientos, en su actitud frente a la vida, en sus aficiones, en su capacidad intelectual.

En un manual de geografía para estudiantes de primero de bachillerato (Valencia de Porras, 1977: 87-88) se lee: 
"En relación con las actividades económicas, especialmente la agricultura podemos decir:

1. Todos los cultivos dependen del clima.

2. En donde la agricultura no es posible 1 por el clima, los habitantes se dedican a la ganadería o a la pesca.

3. Las regiones del mundo que disfrutan de estaciones han logrado gran adelanto, ya que las variaciones estacionales estimulan la previsión del ahorro

Es innegable la influencia del clima en el modo de ser de las personas; en las maneras de sentir, pensar y actuar. En las latitudes altas, por la baja presión atmosférica, el hombre reacciona más lentamente y su pensamiento es tardío. En las regiones de baja latitud, las altas temperaturas, la humedad y las bajas presiones impiden la plena actividad del hombre. En cambio, en las zonas de latitudes medias, de cambios estacionales, el hombre desarrolla una mayor actividad física y mental.

Por lo que hace a Colombia, y como ya lo habíamos estudiado, tenemos que hay diferentes tipos regionales según el paisaje y el clima: dijimos que el hombre del litoral, es alegre, inteligente, amante de la música y el baile. El llanero, dueño de extensiones ilimites, es valeroso, amante de la libertad, arrojado y hospitalario".

En el libro de Soler (1 977: 90) para cuarto de bachillerato se afirma que:

"El clima determina en el hombre la condición de su vivienda y la clase de su vestido.

Los habitantes de ja Región Andina, en su mayoría, pertenecen a los grupos blanco y mestizo. En esta región habitan varios tipos humanos, con características propias, debido al mosaico de paisajes naturales que les imprime su peculiar manera de ser.

La variedad de climas y los diversos aspectos de la topografía andina, han dado lugar a un rico y espléndido folclore que es orgullo del pueblo colombiano.

En otro texto de geografía universal (Marañón et al 1980a: 65) los autores aducen sobre la expansión europea que "esta expansión fue posible por varios factores como su ventajosa posición en el globo; la forma misma del continente y lo accidentado de sus costas, que les permitió el establecimiento de puertos marítimos y la conquista de jos mares". Y en su libro sobre geografía económica de Colombia, los mismos autores (Marañón et al 1 980b: 66) exponen así las influencias del clima:

"Dentro del paisaje geográfico, el clima es uno de los elementos de mayor influencia, por cuanto determina, directamente, la posibilidad que ofrece una región para el establecimiento y la actividad humana. La actividad agropecuaria del hombre de una región depende del tipo de clima que exista.

El tipo de vivienda depende íntimamente del clima, pues en aquellos de alta temperatura, la casa será amplia, ventilada, en oposición a la de clima frío, en donde es reducida y abrigada. Igualmente influye en el vestido, pues en tierras cálidas son de uso común las telas livianas, mientras que en el clima frío son las telas gruesas. 
La alimentación varía de una región a otra, dado que la base del alimento diario es el producido por la región.

La intensidad del trabajo del hombre también depende del clima, ya que en tierras cálidas debido a las altas temperaturas, su labor se hace más difícil limitándose en la mayoría de los casos a la ganadería y a la pesca. En jas regiones de clima medio la intensidad del trabajo del hombre es mayor, pues las condiciones se lo permiten, dedicándose a jas labores agrícolas y pecuarias. En los climas fríos se reduce un poco el grado de actividad, dedicándose en menor escala a la agricultura, ganadería y artesanía.

Al igual que en el caso anterior, el temperamento humano también es influenciado por el clima. La manera distinta de actuar del hombre colombiano, su carácter extrovertido o introvertido (costeño-pastuso), su actitud ante ja vida, sus aficiones, etc., sor una muestra de esa influencia.

Todo lo expuesto hasta aquí deja en claro que en la enseñanza de la geografía en Colombia, las relaciones del hombre con el medio natural se plantean en un esquema causal unilateral, de leyes absolutas, en el que el hombre aparece como un ser fuera de la naturaleza, enfrentado a ella, y en últimas sometido a sus designios, dentro de un rango de posibilidades por demás estrecho. Este enfoque determinista ya ha sido dejado de lado en la geografía moderna, que sin negar la causalidad ni las influencias del medio, examina las relaciones hombre-medio como interacciones complejas y dialécticas. También la geografía ha adoptado los modelos de explicación probabilista. Pero nuestros autores parecen ignorar tales cambios y sus lecciones de geografía se anclaron en el pasado y son poco diferentes, o mejor nada diferentes, de las lecciones de Ritter, Giyot, Ellen Churchill Semple o de Huntington. Parece como si la geografía no hubiera cambiado desde cuando hace casi ciento ochenta años el sabio Caldas concluyera en su estudio sobre el influjo del clima en los seres organizados (Universidad Nacional, 1966: 119), lo que sigue:

"Que se reúnan los efectos del calor y del frío, de la presión atmosférica, de la electricidad, de las montañas, de los vientos, de los ríos, de las selvas, de las lluvias, y de los alimentos; que se acumulen sobre los individuos en diferentes proporciones, y combinados de todos los modos posibles; en fin, que su imperio se perpetúe y pase de generación en generación. Los productos variarán como las causas: el hombre adquirirá el color negro, blanco aceitunado y todas las tintas; su estatura irá desde la gigantesca hasta la pigmea; sus facciones, desde la deformidad hasta la belleza; su moral, desde las virtudes hasta los vicios; y en una palabra, el hombre se modificará en todas sus partes, y cederá a la potencia activa y enérgica del clima.

\section{CONCLUSION}

A estas alturas, ya los ejemplos citados son suficientemente contundentes para afirmar que la enseñanza de la geografía en Colombia ha permanecido estancada y que por lo menos en los últimos treinta años el enfoque determinista se repite de texto en texto, sin que -se asuma una reformulación crítica acorde con el desarrollo de la ciencia geográfica. Tal estancamiento se debe por una parte a la carencia de geógrafos profesionales que asumen la producción de materiales y manuales de enseñanza, ante lo cual la producción de los mismos ha ido a parar en manos ajenas al quehacer geográfico, como las de filósofos, historiadores, sicólogos o de humanistas polígrafos. Por otra parte, no se puede 
eludir la responsabilidad cabida a los departamentos de geografía de las facultades de educación, a las sociedades y asociaciones de geógrafos que han permanecido en silencio ante el catastrófico manejo de la disciplina; y no de últimos, a los profesores de geografía que aunque en su gran mayoría son hoy día Licenciados en Ciencias Sociales, han asumido una actitud acrítica e indiferente, a la vez que han estimulado el uso de tales textos, pues al fin y al cabo, son ellos los que los exigen a sus alumnos.

Una ardua pero vivificante tarea espera a las futuras generaciones colomb ian as de geógrafos profesionales.

\section{REFERENCIAS}

Adamou, Jacqueline (1983), "La Aufklarung. El Romanticismo" en Chatelet, F. (1983) La Filosofta del mundo moderno, Los Ilustrados, cuarta parte, Madrid, Editorial EspasaCalpe.

Arbeláez Lema, Federico (1967), Geografía Universal Americano, Bogotá, Ed. Voluntad.

Broek, Jan O.M. (1965), Geography: Its Scope and Spirit, Columbus, Ohio, Charles: E. Merill Books (trad. por Manuales Uthea, Geografía, Su Ambito y su Trascendencia (1967).

Claval, Paul (1974), Evolución de la geografía humana, Barcelona Oikos-tau.

Estébanez, José (1982), Tendencias y problemática actual de la geografía, Madrid, Ed. Cincel.

Franco R., Ramón (1952), Colombia, Geografía Superior Económica y Humana, Bogotá, imp. Banco de la República.

Franco R., Ramón; Franco B., Fabiola (1982), Geografía Económica de Colombia, Medellín, Ed. Bedout.

Gómez Mendoza, Josefina; Muñoz Jiménez, Julio; Ortega Cantero, Nicolás<1982), E/pensamiento geográfico, Madrid, Alianza Editorial.

Guyot, Arnold (1852), Earth and Mand. Lectures on Comparativa Physical Geography, Boston, Gould and Lincoln.

Harvey, David (1969), Explanation in Geography, New York, St. Martin's Press. (ed. cast. Alianza Editorial, Madrid, (1983) Teorías, Leyes y Modelos en Geografía).

Huntington, Ellsworth (1940), Principles of Human Geography, New York, John Wiley Sons Inc.

Isachenko, A.G. (1971), "Determinism and Indeterminism in Foreign Geography. Par 1", Soviet Geography: Reviewand Traslation, Vol XIII, No. 7,421-431.

James, Preston E. (1972), Ah Possib/e Worlds - A history of geographical ideas, Indianapolis, The Odyssey Press (2a. Ed. Por John Willey, 1981).

Justo Ramón (19858), Geografía Superior de Colombia, 7a. Ed., Bogotá, Colección la Salle.

Lewthwaite, Gordon R. (1966), "Enviromentalism and Determinism: A Search for Clarification",

Annals of the Association of American Geographers, Vol 56 No. 1, March 1966, pp 1-23.

Marañón G., Mortimer; Gómez, Hilda; Vega, Alcira; de López, Gilma; Prada, Cora (1980a), Geografía Universal: Asia, Europa, Africa, Oceanía y Regiones Polares, Bogotá, Ed. Norma.

(1980b), Geografía Económica de Colombia, Bogotá, Ed. Norma.

Ocampo, López; Franco A., Ramón (1968), Geografía Superior de Colombia, Medellín, Ed. Bedout. Soler Delgado, Félix A. (1977), Geografía Económica de Colombia, Bogotá,

Ed. Cultural Colombiana. 
Stoddart, D.R. (1966), "Darwin's Impact on Geography", Annals of the Association of American Geographers, Vol 56, No. 4 Dec. 1966, pp. 683-698.

Universidad Nacional de Colombia, ed (1966), Obras completas de Francisco José de Caldas, Bogotá, Im. Nacional.

Valencia, Pedro; de Porras, Argenis (1977), Geografía General, Física y de Colombia, Bogotá, Ed. Cultural Colombiana.

Vilá Valentí, J. (1983), Introducción al estudio teórico de la geografía, Vol. 1., Barcelona, Ed. Ariel. 